\title{
Análisis del Desgaste Para el Par Bronce - Acero en un Molino de Caña de Azúcar
}

* Msc., Profesor - Escuela de Ingeniería Mecánica - Facultad de Ingeniería - Universidad del Valle, Santiago de Cali, Colombia.

e-mail: johncoro@petecuy.univalle.edu.co
Fecha de recepción: Septiembre 10 de 2004

Fecha de aprobación: Diciembre 17 de 2004

\author{
John Jairo Coronado Marín*
}

\section{RESUMEN}

Los ejes y las chumaceras de molino de caña de azúcar sufren excesivo desgaste en condiciones normales de operación. En este estudio se efectuaron observaciones metalográficas y de microscopía electrónica de barrido para identificar el mecanismo de desgaste del par bronce-acero. Se encontró que las marcas dejadas sobre la superficie del eje por partículas abrasivas, disminuyen su resistencia a la fatiga y favorecen el crecimiento de fisuras superficiales. Las fisuras se propagan en forma de fisuras semilípticas o circunferenciales. El sobre-calentamiento de las chumaceras produce segregación y fusión del plomo presente en el bronce, permitiendo la formación y propagación de las fisuras. 
Palabras Clave: Eje, chumacera, desgaste, fisuras.

\section{ABSTRACT}

The shaft and the bearing of sugar cane mill undergo excessive wear under normal operation conditions. Optical microscope and scanning electron microscope were used to characterize surface phenomena in the tribological pair steelbronze. Abrasive particle marks were founded on the surface of the shaft; they reduce the fatigue strength, since they induce the superficial crack growth. The cracks grow in a semilliptical or circumferential shape. Over heating of the bearing do produce lead segregation and fusion, promoting the cracks formation and propagation.

Key Words: Shaft, bearing, wear, cracks.

\section{INTRODUCCIÓN}

La extracción del jugo presente en la caña se realiza usando un tren de molienda que está constituido por varios molinos, los cuales extraen jugo uno tras otro hasta que la caña preparada se reduce a bagazo. En la Figura 1 se muestra el eje típico de un molino de caña de azúcar, sobre éste se encaba la maza por diferencia de temperaturas. La aplicación de la fuerza sobre el eje se realiza por cilindros óleo neumáticos. Los ejes se apoyan sobre cojinetes de bronce más conocidos como chumaceras, ver figura 1, los ejes tienen la posibilidad de flotar para ajustarse al cambio de molienda y evitar que se fracturen debido a una variación brusca de carga. La velocidad de rotación del eje normalmente está entre 4 y 7 rpm y funcionan durante 24 horas al día en los Ingenios Colombianos.

Los molinos de caña de azúcar habitualmente están contaminados con una gran cantidad de materia extraña de origen mineral y orgánica, que viene con los tallos de la caña. La caña trae parte del suelo, que habitualmente está compuesto por sílice o cuarzo (arena), estos componentes entran en el guijo, que es la parte del eje que se apoya en la chumacera (Figura 1). Esto puede provocar desgaste abrasivo afectando directamente la superficie y dando la posibilidad de inicio de grietas superficiales. El cambio de diámetro del eje hace que el hombro se convierta en un concentrador de esfuerzos y por esta razón los ejes fallan en gran proporción en esta zona. Los molinos de caña tienen como principio el aplicar grandes presiones a la caña a bajas velocidades de rotación, con el fin de extraer el jugo que ésta contiene, por tal motivo los ejes se encuentran sometidos a estados de esfuerzo de flexo- torsión.

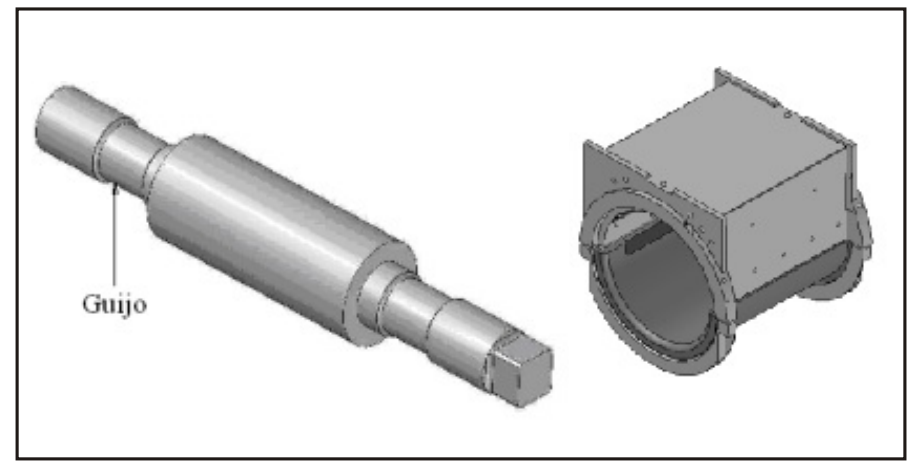

Figura 1. Eje y chumacera de molino de caña de azúcar

\section{OBSERVACIÓN MACROSCÓPICA Y MICROSCÓPICA DEL EJE}

El análisis visual de las superficies desgastadas muestra marcas típicas del desgaste abrasivo por el contacto con partículas duras. Cuando se presenta la flotación del eje la chumacera se levanta permitiendo el ingreso del jugo de la caña con materia extraña favoreciendo el desgaste del par eje-chumacera. La formación de surcos en el eje y la chumacera debido a materia extraña se muestra en la figura 2 .

La figura 3 muestra la superficie desgastada del eje a diferentes aumentos, donde se aprecia la formación de surcos en el sentido de rotación del eje. En la figura se pueden apreciar diferentes 
profundidades y ancho de huella dejada por el paso del abrasivo, lo cual indica que las partículas abrasivas son de diferente tamaño. El ancho de la huella en el eje está en un rango entre 100 y 300 $\mu \mathrm{m}$, también se encontraron marcas entre de 8 y $40 \mu \mathrm{m}$. Además, se presenta deformación plástica del material recubriendo los surcos, debido al movimiento axial del eje sobre la chumacera por el excesivo juego, cuando los elementos en contacto se han desgastado.



Figura 2. Marcas de desgaste abrasivo en el eje y la chumacera

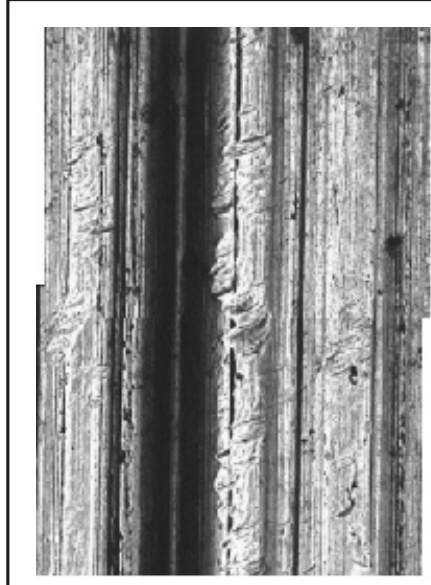

(a)

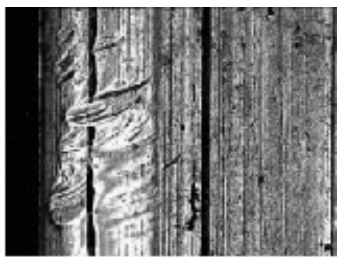

(b)

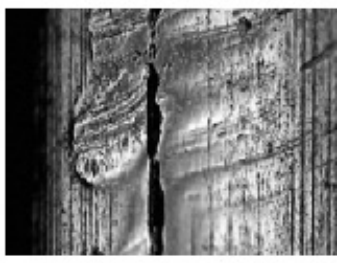

(c)
Figura 3. Ampliación de las marcas de desgaste en el eje (a) 50X; (b) 100X; (c) 200X

En la figura 4 se muestra la microscopía electrónica de barrido de las partículas abrasivas que vienen con la caña y entran en contacto con la superficie del eje y la chumacera. En el molino 1 se encontró un diámetro promedio de $130 \mu \mathrm{m}$ y en el molino 6 fue de $113 \mu \mathrm{m}$, debido a que las partículas se fracturan por la presión hidráulica al pasar por tren de molinos (Gordillo, 2004). Resultados de otros molinos presentaron un diámetro promedio en el primer molino de 237 $\mu$ m y en el molino 6 se encontraron tamaños con frecuencia de repetición similar entre 125 y 170 $\mu \mathrm{m}$. De acuerdo a los datos anteriores se puede concluir que las partículas abrasivas que vienen con la materia extraña producen marcas en el eje y la chumacera entre 100 y $300 \mu \mathrm{m}$. Debido al desgaste adhesivo fragmentos de acero y de bronce desprendidos y endurecidos por deformación plástica, quedan sobre la superficie de contacto, los cuales dejan marcas de desgaste en un rango entre 8 y $40 \mu \mathrm{m}$.



Figura 4. Microscopia electrónica de barrido de partículas abrasivas típicas en los molinos 1 y 6 respectivamente.

En los picos de los surcos se pueden observar indicaciones de desgaste adhesivo por la presión específica que se alcanza en esos puntos. Cuando las superficies se oprimen entre ellas, algunas de las asperezas en contacto tienden a adherirse recíprocamente por fuerzas de atracción entre átomos superficiales en ambos materiales. La figura 5 muestra la superficie de desgaste del eje usando microscopio electrónico de barrido, donde se evidencia desgaste adhesivo del eje de acero. Se puede apreciar el arrastre del material que se ha desprendido, ya que durante el deslizamiento las superficies ásperas experimentan deformación y fractura; ya sea a través de la interfaz original o a lo largo de un nuevo plano. Las partículas desprendidas quedan como un desecho en la interfaz que raya la superficie y labra surcos en las dos piezas.

Después de estudiar probetas de acero SAE 
1045, en una máquina de fatiga de flexión rotatoria, sumergidas en jugo de caña con Ph de 4 (Gómez, 2004), se encontró una disminución del $11 \%$ en el límite a fatiga del material de los ejes. El mecanismo de desgaste de los ejes se puede considerar como una combinación de desgaste abrasivo, adhesivo y corrosivo.

El análisis químico del acero del eje indica que el contenido medio de carbono es de 0.45, es decir que corresponde a un acero SAE 1045. Para el análisis metalográfico se montaron las probetas en resina, se usaron lijas con granulometría de $240,320,400$ y 600. Para la operación de pulido se utilizó óxido de aluminio de $0.3 \mu \mathrm{m}$ y el reactivo usado fue nital al $2 \%$. En la figura 6 , se muestra la microestructura del eje que consiste de granos de perlita de color oscuro y ferrita de color blanco. Esta microestructura confirma los resultados obtenidos en el análisis químico. De acuerdo a lo observado en la microestructura no se le efectúo ningún tratamiento térmico o recubrimiento al acero, después del proceso de forjado.

En la micrografía de la figura 7 se observa una discontinuidad que se podría tratar de una inclusión no metálica, que es un concentrador de esfuerzos, generando fisuras bajo la superficie, a lo largo de una banda de alto contenido de ferrita.

En la figura 8 se puede apreciar el grano deformado plásticamente, debido al paso sucesivo de partículas abrasivas sobre la superficie del eje. El mayor desgaste se presenta como consecuencia de la deformación del material en la superficie del eje en el sentido opuesto al giro del mismo. Si las partículas están incrustadas en el cojinete, en su superficie de trabajo mostrará puntos discontinuos de contacto que son los puntos del cojinete deformados por la incrustación de las partículas. En muchos casos y a pesar de la mayor dureza del eje, éste sufre un fuerte desgaste por la retención de las partículas en el cojinete y el consecuente trabajo mecánico sobre el eje. En la Figura 9 se muestra la representación esquemática de la formación de surcos, debido al paso de una partícula abrasiva, produciendo deformación plástica en los bordes del surco.



Figura 5. Microscopía electrónica de barrido de la superficie del eje

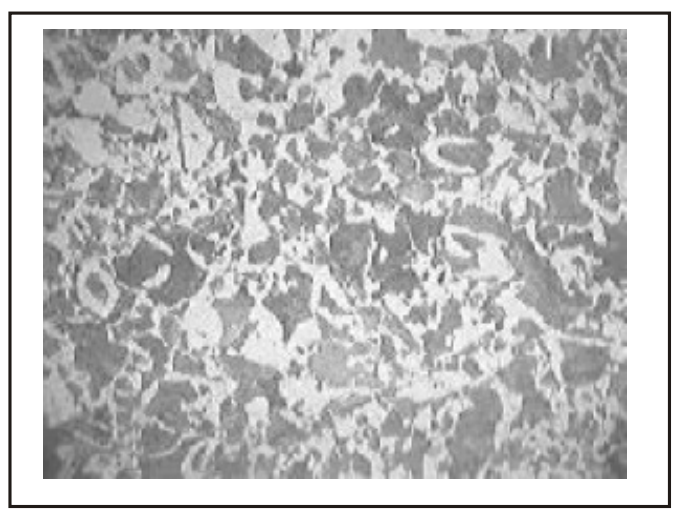

Figura 6. Microestructura longitudinal del eje a $250 \mathrm{X}$

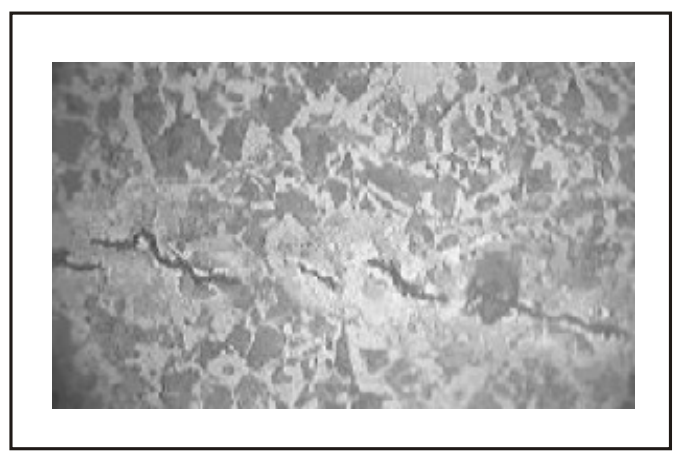

Figura 7. Imperfección microestructural en el eje de acero 


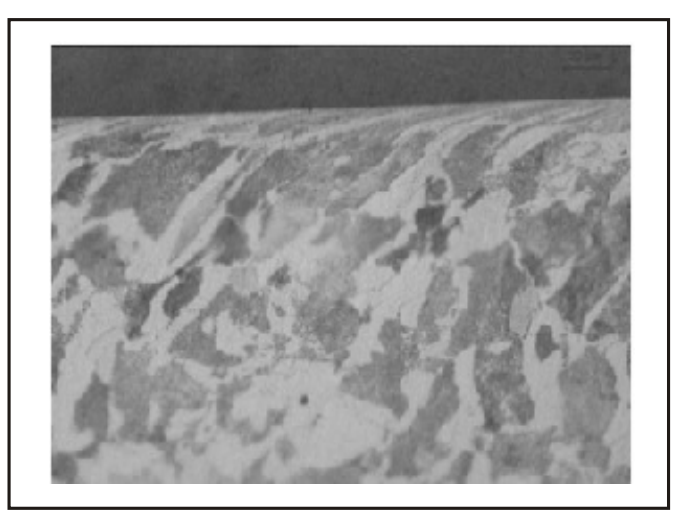

Figura 8. Deformación plástica en la sección transversal del eje

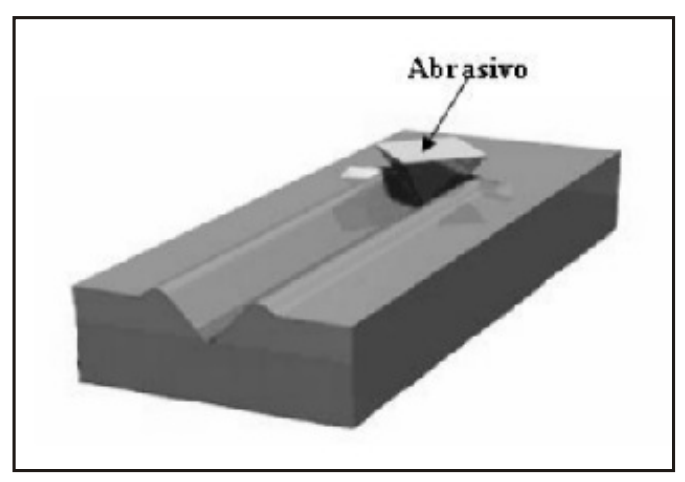

Figura 9. Representación esquemática de la formación de surcos

La causa más frecuente del desgaste abrasivo es la contaminación del lubricante con partículas duras, bajo la influencia de una fuerza. Cuando se presenta la flotación del eje por carga no uniforme de caña, ingresa materia extraña. Teniendo en cuenta que el espesor de la película de aceite es de 5 a 10 micrones, partículas duras de mayores dimensiones arrastradas por el sistema de lubricación, producirán deterioros. En ciertos materiales (por ejemplo los metales blandos) las partículas duras tienden a incrustarse en el cojinete y si su tamaño es demasiado grande emergen de la superficie de trabajo deteriorando al eje, el cojinete se convierte en un verdadero portaherramientas. La formación de surcos se presenta sobre la superficie del eje en el sentido transversal del desgaste. Rayas y surcos paralelos a la dirección del movimiento del eje indican el patrón del desgaste. Usualmente los surcos están bien definidos y fueron producidos por la deformación y desplazamiento del material más que por la remoción del mismo. En la Figura 10 se aprecia claramente una fisura que se inició sobre una marca de desgaste abrasivo, la fisura se cierra sobre la superficie del eje debido a la deformación plástica que experimenta la superficie del eje por el paso continuo de partículas abrasivas.

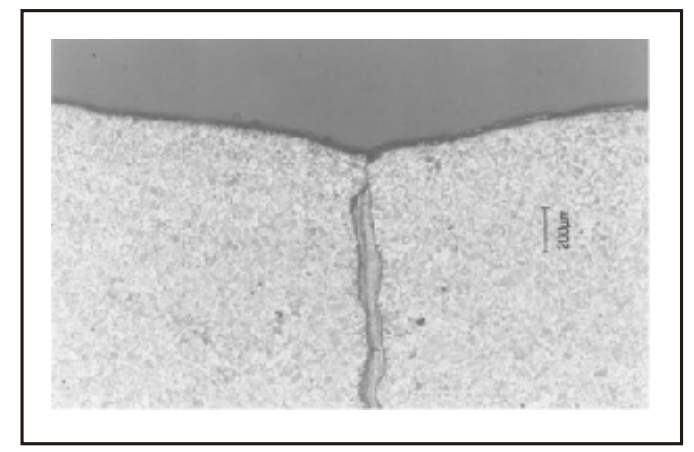

Figura 10. Micrografía transversal a las marcas de desgaste

Los surcos o marcas dejadas por partículas duras disminuyen la resistencia a la fatiga del eje. Estos surcos aumentan la concentración de esfuerzos provocando la formación de fisuras que se van propagando en varios puntos en forma de fisuras semielípticas, hasta que se unen para formar fisuras circunferenciales. El material remanente no puede soportar las cargas y se presenta la fractura del eje. En la figura 11 se muestra la superficie de fractura de un eje que falló debido a la propagación de una fisura circunferencial, se observan las marcas de playa en la superficie de fractura del eje típica de una falla por fatiga. La fisura circunferencial se presenta debido a varios concentradores esfuerzos (marcas de desgaste) a lo largo del perímetro del eje. En la Figura 12 se muestra la superficie de fractura de un eje que falló debido a la propagación de fisuras semielipticas hasta que el eje remanente no puede soportar la carga aplicada y se presenta la fractura final. 


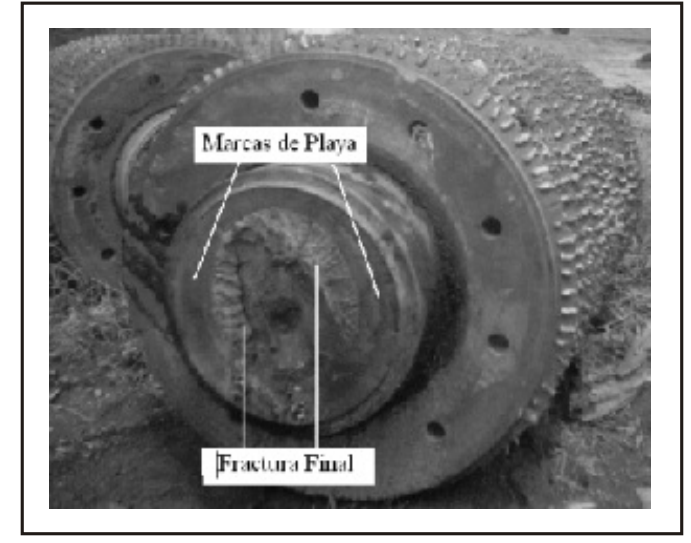

Figura 11. Fractura provocada por una fisura circunferencial

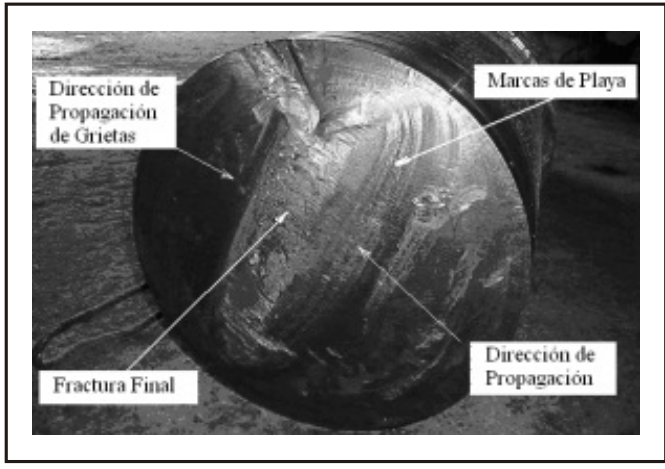

Figura 12. Fractura provocada por fisuras semielípticas

\section{OBSERVACIÓN MACROSCÓPICA Y MICROSCÓPICA DE LA CHUMACERA}

El análisis de los elementos de aleación de la chumacera de bronce usando análisis gravimétrico y por absorción atómica, arrojó los siguientes resultados: estaño $7.5 \%$, plomo $9.2 \%$, zinc $0.5 \%$ y cobre $84.8 \%$. El porcentaje de plomo está muy bajo, ya que para un acero SAE 67 usado como material para chumaceras, por sus buenas propiedades mecánicas, debe tener entre 14 y $18 \%$ de plomo. El bajo contenido de plomo aumenta la dureza de la chumacera y disminuye las propiedades lubricantes del bronce. Al realizar el análisis metalográfico de las probetas de bronce, se encontró una distribución no uniforme del plomo sobre la matriz. Existen zonas con muy poco contenido de plomo y otras con gran concentración de plomo, como se observa en la figura 13.ayb.



Figura 13. Distribución del plomo en el bronce a $250 x$

En el eje y la chumacera se presentan elevadas temperaturas debido a la falta de refrigeración continua, a la fricción generada por la lubricación límite y a las elevadas presiones a las que está sometido el par. Cuando se presentan dichos sobrecalentamientos en la chumacera, el plomo se segrega y se funde (Figura 14), favoreciendo la formación y propagación de fisuras.

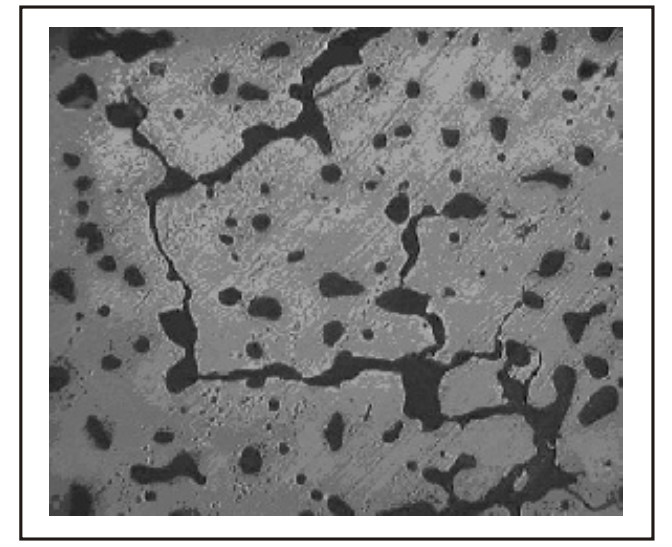

Figura 14. Plomo segregado en el bronce a $250 x$

En las figura 15 se puede observar a simple vista fisuras sobre la superficie de la chumacera, generadas en quemaduras dejadas por sobrecalentamiento del material

La figura 16 presenta la microscopía electrónica de barrido sobre la superficie desgastada de la chumacera, que indica desgaste adhesivo. Se 
evidencia el desprendimiento más severo en el bronce, estos fragmentos posteriormente rayan la superficie de los elementos en contacto.

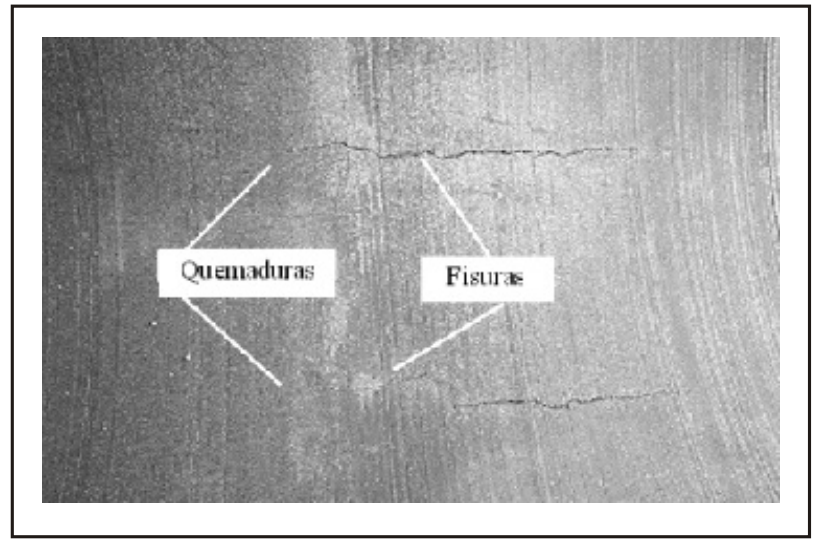

Figura 15. Superficie fisurada de la chumacera

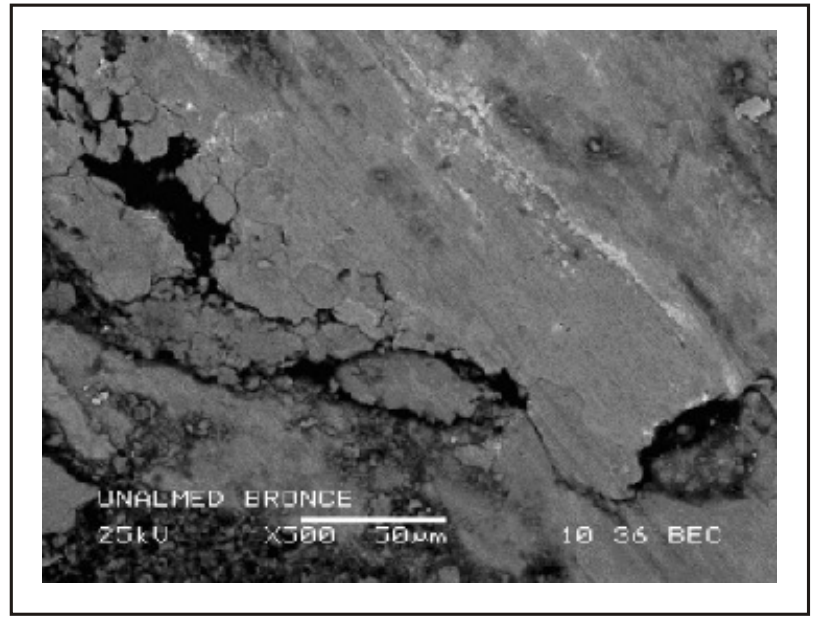

Figura 16. Microscopía electrónica de barrido sobre la superficie de la chumacera

\section{DUREZAS}

Se encontró que los ejes sin recuperar tienen una dureza comprendida en un rango entre 180 y 220 BHM y para las chumaceras la dureza se encuentra entre de 60 y 90 BHM. Es decir, la razón de durezas está entre 2.0 y 3.7. Estudios sobre el comportamiento del par acero con bronce al estaño o bronce al plomo recomiendan una razón de durezas de 5 (Wilcock, 1957; Morton 1999).
Una relación de durezas tan baja aumenta el desgaste del eje, que es un elemento mecánico muy costoso, la chumacera debe ser el elemento de sacrificio.

\section{CONCLUSIONES Y RECOMENDACIONES}

El recubrimiento de los surcos a través del movimiento axial del eje muestra que existe juego en el sistema, debido al desgaste del par bronce-acero, perjudicando la operatividad del conjunto.

El eje presenta elevado desgaste sobre la superficie de contacto con la chumacera generando problemas operacionales. Estas marcas de desgaste indican una deficiente lubricación del par. La formación de surcos profundos indica la presencia de materia extraña como por ejemplo la arena. Se recomienda lavar la caña antes de pasar por el proceso de preparación y molienda, para disminuir la materia extraña que viene con la caña.

La microestructura del eje es ferrita y perlita, sin tratamiento superficial como la cementación o la metalización. Tratamientos superficiales pueden aplicarse para mejorar el rendimiento del conjunto.

La vida útil del eje puede ser aumentada con un sistema de sellado de la chumacera para evitar el ingreso de materia extraña.

Se debe usar un bronce con una dureza máxima 60 BHM y un acero con una dureza mínima de 300 BHM para garantizar una relación de durezas de 5 a 1 .

Se deben garantizar una adecuada refrigeración del sistema, ya que sobrecalentamientos producen segregación y fusión del plomo, situación que favorece la formación y propagación de fisuras. 


\section{AGRADECIMIENTOS}

El Grupo de Investigación en Mejoramiento Industrial, de la Escuela de Ingeniería Mecánica (Universidad del Valle), en el marco del "Proyecto Cooperativo Agroindustrial para Reducir el Desgaste en Equipos de Preparación y Molienda" que se lleva a cabo en el Ingenio Manuelita S.A., con la participación de CEMICAÑA, agradece los aportes del personal de estas organizaciones y el apoyo financiero de COLCIEMCIAS, que con dinero del BID, ha posibilitado este trabajo. También se agradece a Alejandro Toro de la Universidad Macional de Colombia, Sede Medellín, por permitir el uso del Laboratorio de Microscopía Avanzada.

\section{REFERENCIAS}

[1] ASM Handbook. "Friction, Lubrication, and Wear Technology" Volume 18. 1992.

[2] Wilcock and Booser. "Design and Application". Mc Graw Hill. 1957.

[3] Morton R.L. "Diseño de máquinas". Prentice Hall. 1999.

[4] Radzimovsky E.I. "Lubrication of Bearings". Ronald Press M.Y. 1959.

[5] Bhushan B. "Principles and applications of Tribology" John Wiley \& Sons, Inc. 1999.

[6] Gordillo D. "Relación entre la materia mineral abrasiva en los molinos de caña y el desgaste abrasivo". Universidad del Valle, Cali. 2004.

[7] Gómez J. A. "Análisis del comportamiento a fatiga de un acero para ejes en ambiente corrosivo". Universidad del Valle, Cali. 2004.

[8] Coronado, J. J., Rivas, J. S., Gómez A. L. "Estudio tribológico en chumaceras y ejes de Molino de caña de azúcar" Dyna. Mo 144. Pp. $1-8,2004$. 